\title{
Algebraic properties of external numbers
}

\author{
BRUNO DINIS \\ IMME VAN DEN BERG
}

\begin{abstract}
Neutrices and external numbers were proposed as models of orders of magnitude within nonstandard analysis. We show that the external numbers form a commutative regular semigroup for addition and that the external numbers which are not neutrices form a commutative regular semigroup for multiplication. The validity of the distributive law is restricted, but it can be fully characterized.
\end{abstract}

2000 Mathematics Subject Classification 03H05 (primary); 06F05, 20M14, 20M17 (secondary)

Keywords: neutrix, external number, regular semigroup, distributivity, nonstandard analysis

\section{Introduction}

External numbers were introduced in [16] and [17], as mathematical models of orders of magnitude within nonstandard analysis. In the present paper we study the external numbers from an algebraic point of view. Within the external numbers we distinguish neutrices, a sort of generalized zeros (see below). We show that the external numbers form a commutative regular semigroup for addition and that the external numbers which are not reduced to neutrices form a commutative regular semigroup for multiplication. Although the operations are not connected by a complete distributive law, we give necessary and sufficient conditions for distributivity to hold. We also prove in an algebraic way some results which in [16] and [17] were proved by set-theoretic arguments.

A neutrix is an additive convex subgroup of $\mathbb{R}$ and an external number is the algebraic sum of a real number with a neutrix (we use an axiomatic approach in which all infinite standard sets have nonstandard elements, so most neutrices are external sets). Being stable for some translations, additions and multiplications, external numbers are models of orders of magnitude or transitions with imprecise boundaries. In the nonstandard framework there are many neutrices, enabling to solve paradoxes which arise when 
several orders of magnitude are simultaneously considered. These paradoxes are called Sorites paradoxes. One can be stated in the following way: a single grain of wheat cannot be considered as a heap. Neither can two grains of wheat. One must admit the presence of a heap sooner or later, so where to draw the line? In fact, the heap and the grain of wheat are not of the same order of magnitude and we might say that the set of individual grains may be modeled by the external set of limited numbers (positive part of a neutrix) and the set of grains that form a heap may be modeled by the external set of the infinitely large numbers. It should also be possible to capture in this way some modalities, like the difference between a "good" approximation, allowing to obtain an adequately precise numerical result in some context, and a "bad", useless, one.

The stability of orders of magnitude under some repeated additions justifies to model them by (convex) groups of real numbers. Historically, the term neutrix was first used by Van der Corput [6], referring to groups of functions. Among others, his objective was to deal with imprecisions arising from neglecting terms of expansions. There are other approaches to this kind of problems, such as the $o$ and $O$ notation [4], confidence intervals of statistics, interval arithmetic [10] [18] and fuzzy sets [22]. These other approaches are not without fault as models of imprecisions, because they ultimately recourse to precise intervals to model imprecise situations, and do not work with the actual error but only with an upper bound of the error. On the contrary, with external numbers it is possible to work directly with imprecisions and errors without recourse to upper bounds, for they have neither infimum nor supremum and satisfy the algebraic laws mentioned above. Moreover, the external numbers are totally ordered, even allowing for a sort of generalized Dedekind completeness property [1] [2] [17]. In the context of model theory [15] considers two other completeness properties of the external real line, Scott completeness and Bolzano-Weierstrass completeness, reconsidered from an axiomatic point of view in [13].

It had been recognized by Wattenberg [21] and Gonshor [9] that it is possible to develop a calculus on external Dedekind cuts, to be more precise the lower halflines. However they obtained a less rich algebraic structure, than it was possible to identify with external numbers.

This article has the following structure. In Section 2 we discuss some foundational aspects concerning external sets which are relevant for the construction of neutrices and external numbers as well as sets of neutrices and external numbers.

In Section 3 we recall the notions of neutrix and external number, the definitions of sum, multiplication and order of external numbers and some elementary properties.

In Section 4 we first recognize the set of external numbers when equipped with addition as a regular semigroup and, leaving out the neutrices, as a regular semigroup for 
multiplication. This allows us to obtain individualized neutral and inverse elements for the defined operations, and to define a structure called assembly which in some sense generalizes the notion of group. Then we study some algebraic properties of assemblies, in particular a cancellation law. Finally we study mixed properties of addition and multiplication of external numbers, such as the absence of zero divisors.

In the final section we show that the distributive law does not always hold. We give sufficient and necessary criteria for the distributive law to be true. We illustrate the criteria with some examples and applications, in particular we give a condition for the binomial law to hold.

We thank Ulf Clotz (University of Wuppertal) for many enlightening discussions on foundational matters with respect to external sets, and in particular, external numbers.

\section{Foundational aspects}

As was observed in the introduction neutrices and external numbers essentially are external sets. In the context of model theory a nonstandard model of the real number system generates neutrices in an obvious way, since ${ }^{*} \mathbb{R}$ contains external convex subgroups. It is convenient to choose a superstructure in the sense of Robinson-Zakon [20][23], to be able to apply the usual operations of analysis on neutrices and external numbers. It is also convenient to suppose the superstructure to be $k$-saturated, for some infinite cardinal $k$. Then neutrices of the lowest complexity, i.e. neutrices of the form $\cup_{x \in X}\left[-a_{x}, a_{x}\right]$ and $\cap_{x \in X}\left[-a_{x}, a_{x}\right]$, where $X$ is a (standard) set of cardinality less than or equal to $k$, satisfy some strong properties. Indeed, it is not difficult to adapt the proofs of axiomatic nonstandard analysis in [2][17] to derive firstly that every neutrix $N$ of this form is the multiple $N=\lambda I$ by a hyperreal number $\lambda$ of an idempotent neutrix $I$ (i.e., with $I . I=I$ ) and secondly that every external lower halfline of ${ }^{*} \mathbb{R}$ is bounded from the above by an external number, which is cofinal with it, or just beyond.

With respect to axiomatic nonstandard analysis more care is needed. The axiomatics IST of Nelson [19] formulates within first-order language the behaviour of standard and internal sets of a (strong) nonstandard model; external sets are lacking. As argued in [13][14], the axiomatics IST must be modified in order to admit an extension to external sets in a reasonable way. This is done by postulating a boundedness axiom that says that every set belongs to a standard set

$$
\forall x \exists^{s t} y(x \in y),
$$


and since this contradicts Idealization the following form is taken instead:

$$
\forall^{s t} Y\left[\forall^{s t f i n} Z \exists y \in Y \forall z \in Z \varphi \Leftrightarrow \exists y \in Y \forall^{s t} z \varphi\right],
$$

for every $\in-$ formula $\varphi$. This yields the subsystem $B S T$, which corresponds to the bounded sets of $I S T$. Notice that in usual analysis all sets are bounded.

The theory BST possesses an extension to $H S T$ [13][14], which formulates within first-order language essential aspects of the behaviour of standard, internal and external sets within a nonstandard model, much as in Hrbacek's original system [11]. The theory $H S T$ offers the axiom of Separation in the full language and thus deals with external sets. It is possible to maintain the notation of IST in the way that the traditional symbols for the uniquely defined objects of a ZFC universe are affixed to the objects of the internal subuniverse. Each neutrix, being a convex subgroup of $\mathbb{R}$, is a genuine set within HST . Neutrices are defined by $\Pi^{s t}$ or $\Sigma^{s t}$ formulae, with reference to standard sets of all possible cardinals [2]. As shown in [13] this implies that we may not speak of the set of all neutrices. In fact, the neutrices form a definable class because quantification within HST ranges over the whole universe. Then the algebraic operations on neutrices and external numbers are also definable classes, as well as the function which associates to each external number its neutrix.

\section{Preliminaries}

For a thorough introduction to external numbers with proofs we refer to [16] and [17]. A neutrix is an additive convex subgroup of $\mathbb{R}$. Except for $\{0\}$ and $\mathbb{R}$ itself all neutrices are external sets (with internal elements). The most obvious neutrices are $£$, the external set of all limited numbers and $\oslash$, the external set of all infinitesimal numbers. There are many neutrices not isomorphic by internal homomorphisms [2][3]. All neutrices are external sets of the form $\cup_{x \in X}\left[-a_{x}, a_{x}\right]$ or $\cap_{x \in X}\left[-a_{x}, a_{x}\right]$, where $X$ contains only standard elements (or is of standard size) and $a: X \rightarrow \mathbb{R}$. We denote the external class of neutrices from now on by $\mathcal{N}$.

Addition and multiplication on $\mathcal{N}$ are defined by the Minkowski operations. So, if $A$, $B$ are neutrices we define their sum by

$$
A+B=\{a+b \mid(a, b) \in A \times B\}
$$

and their product by

$$
A B=\{a b \mid(a, b) \in A \times B\} .
$$

Neutrices are ordered by inclusion. The sum of two neutrices is the larger of the two: 
Proposition 3.1 If $A, B \in \mathcal{N}$ then $A+B=\max (A, B)$.

An external number $\alpha$ is the algebraic sum of a real number $a$ with a neutrix $A$. The external class of external numbers will be denoted by $\mathbb{E}$. If $\alpha=a+A$ and $\beta=b+B$ are two such external numbers, the Minkowski sum and product are given by

$$
\begin{aligned}
\alpha+\beta & =a+b+A+B \\
\alpha \beta & =a b+a B+b A+A B .
\end{aligned}
$$

Notice that by Proposition 3.1

$$
\begin{aligned}
\alpha+\beta & =a+b+\max (A, B) \\
\alpha \beta & =a b+\max (a B, b A, A B) .
\end{aligned}
$$

If $\alpha=a+A$ is an external number, then $A$ is called the neutrix part of $\alpha$ and is denoted $N(\alpha)$. An external number which is not a neutrix is called zeroless. One defines $-\alpha=-a+A$ and $1 / \alpha=1 /(a+A)$, if $\alpha$ is zeroless.

Neutrices are stable under multiplication by appreciable numbers, i.e. limited numbers which are not infinitesimal.

Definition 3.2 Let $A$ be a neutrix and $\alpha$ be an external number. We say that $\alpha$ is appreciable with respect to $A$ if $\alpha A=A$, and that $\alpha$ is an absorber of $A$ if $\alpha A \subset A$. N.B. We use the symbol $\subset$ to indicate strict inclusion.

Note that numbers which are appreciable with respect to $\oslash$ or $£$ are simply appreciable.

Proposition 3.3 Let $\alpha=a+A$ be a zeroless external number. Then $\frac{A}{a}=\frac{A}{\alpha} \subseteq \oslash$.

Proof If $x \in \frac{A}{a}$, then $x$ cannot be appreciable, otherwise $a \in \frac{1}{x} A=A$, contradicting the fact that $\alpha$ is zeroless. Hence $\frac{A}{a} \subseteq \oslash$. Moreover $\frac{\alpha}{a}=1+\frac{A}{a}$. Hence $\frac{\alpha}{a} \subseteq 1+\oslash$. So $\frac{a}{\alpha} \subseteq 1+\oslash$. Therefore $\frac{A}{\alpha}=\frac{A}{a} \frac{a}{\alpha}=\frac{A}{a}$.

As a consequence, if $a \notin A$

$$
\frac{1}{1+\frac{A}{a}}=1+\frac{A}{a} .
$$

We state here some elementary properties of the multiplication. The first property is a direct consequence of the definition of multiplication.

Lemma 3.4 Let $\alpha=a+A$ be zeroless. Then $\alpha B=a B+A B$ for all $B \in \mathcal{N}$. 
Lemma 3.5 Let $\alpha=a+A$ and $\beta=b+B$ be zeroless external numbers. Then $\alpha \beta=a b+\max (a B, b A)$.

Proof Since $B \subseteq \oslash b$ by Proposition 3.3, $\max (B A, b A)=b A$. Hence $\alpha \beta=$ $a b+\max (a B, b A, B A)=a b+\max (a B, b A)$.

Lemma 3.6 Let $\alpha=a+A$ and $\beta=b+B$ be zeroless. Then $\alpha \beta=\alpha b+\alpha B$.

Proof Using Lemma 3.4 and 3.5, we derive

$$
\begin{aligned}
\alpha b+\alpha B & =(a+A) b+a B+A B \\
& =a b+b A+a B+A B \\
& =\alpha \beta .
\end{aligned}
$$

Let $\alpha$ and $\beta$ be two external numbers. Then $\alpha \subseteq \beta, \alpha \supseteq \beta$ or $\alpha \cap \beta=\emptyset$. An order relation on $\mathbb{E}$ is given by the following.

Definition 3.7 Given $\alpha, \beta \in \mathbb{E}$, we say that $\alpha$ is less than or equal to $\beta$ and we write (with abuse of notation) $\alpha \leq \beta$, if and only if

$$
(\forall x \in \alpha)(\exists y \in \beta)(x \leq y) .
$$

We say that $\alpha$ is less than $\beta$ and write $\alpha<\beta$, if $\alpha \leq \beta$ and $\alpha \cap \beta=\emptyset$.

Note that if $\alpha \cap \beta=\emptyset$, formula (1) is equivalent to $(\forall x \in \alpha)(\forall y \in \beta)(x<y)$.

Theorem 3.8 [16]The order relation $\leq$ is a total order relation compatible with addition and multiplication in the following way:

(1) $\forall \alpha \forall \beta \forall \gamma(\alpha \leq \beta \Rightarrow \alpha+\gamma \leq \beta+\gamma)$.

(2) $\forall \alpha \forall \beta(N(\alpha) \leq \alpha \wedge N(\beta) \leq \beta \Rightarrow N(\alpha \beta) \leq \alpha \beta)$.

(3) $\forall \alpha \forall \beta \forall \gamma(N(\beta) \leq \beta \leq \alpha \wedge N(\gamma) \leq \gamma \Rightarrow \beta \gamma \leq \alpha \gamma)$. 


\section{Algebraic properties for addition and multiplication}

We start by showing that external numbers when equipped with addition and zeroless external numbers when equipped with multiplication are regular commutative semigroups. In fact, they share more algebraic properties; we will call the structures in question assemblies. The neutral and unit element appear in the form of external functions. We study these functions in some detail, also in order to obtain cancellation laws.

We give algebraic proofs of some properties of addition and multiplication, which were originally proved in [16] by set theoretical arguments.

\subsection{External numbers and regular semigroups}

We recall that a semigroup is a structure that consists of a non-empty set $S$, together with an associative binary operation "*". A regular semigroup is a semigroup $S$ such that every element is regular, that is, for every $a \in S$ there is $x \in S$ such that $a x a=a$. It follows from classical interval calculus that the structures $(\mathbb{E},+)$ and $(\mathbb{E}, \cdot)$ are commutative semigroups. We shall prove that the semigroups $(\mathbb{E},+)$ and $(\mathbb{E} \backslash \mathcal{N}, \cdot)$ are regular.

Theorem 4.1 The structures $(\mathbb{E},+)$ and $(\mathbb{E} \backslash \mathcal{N}, \cdot)$ are commutative regular semigroups.

Proof We need only to prove the regularity properties. Let $\alpha=a+A$ be an arbitrary external number. Put $\delta=(-a+A)$ and $\zeta=\left(\frac{1}{a}+\frac{A}{a^{2}}\right)$. Then

$$
\alpha+\delta+\alpha=(a+A)+(-a+A)+(a+A)=(a+A)=\alpha .
$$

If $\alpha$ is zeroless, necessarily $a \neq 0$, so applying Lemma 3.5

$$
\begin{aligned}
\alpha \zeta \alpha & =(a+A)\left(\frac{1}{a}+\frac{A}{a^{2}}\right)(a+A)=\left(1+\frac{a A}{a^{2}}+\frac{A}{a}\right)(a+A) \\
& =\left(1+\frac{A}{a}\right)(a+A)=a+A+\frac{a A}{a}=a+A=\alpha .
\end{aligned}
$$

Hence $(\mathbb{E},+)$ and $(\mathbb{E} \backslash \mathcal{N},$.$) are commutative regular semigroups.$

\subsection{Properties of neutral and inverse elements; assemblies}

Uniqueness for identity and inverse elements holds neither for addition nor for multiplication, as seen by the following examples. 
Example 4.2 Let $\alpha=1+\oslash$ and $\varepsilon \simeq 0$.

(1) $\alpha+\oslash=1+\oslash+\oslash=1+\oslash=\alpha$ and $\alpha+\varepsilon \oslash=1+\oslash+\varepsilon \oslash=1+\oslash=\alpha$.

(2) Let $\beta=1+\varepsilon \oslash$. Then $\alpha \alpha=(1+\oslash)(1+\oslash)=1+\oslash=\alpha$ and $\alpha \beta=$ $(1+\oslash)(1+\varepsilon \oslash)=1+\oslash+\varepsilon \oslash=1+\oslash=\alpha$.

We introduce functions which generalize the concept of neutral element in order to have individualized neutral elements for addition and multiplication. For $\alpha \in \mathbb{E}$ these functions give us the maximal elements that leave $\alpha$ invariant; as such, they are unique neutral elements. We investigate the properties of such functions and will see that addition and multiplication have a common structure, which leads to the notion of assembly.

Proposition 4.3 There is a unique function $e: \mathbb{E} \rightarrow \mathbb{E}$ such that (i) $\alpha+e(\alpha)=\alpha$ for all $\alpha \in \mathbb{E}$ and (ii) if $f: \mathbb{E} \rightarrow \mathbb{E}$ is such that $\alpha+f(\alpha)=\alpha$ for all $\alpha \in \mathbb{E}$, then $e(\alpha)+f(\alpha)=e(\alpha)$. In fact, $e(\alpha)=N(\alpha)$.

Proof We prove that $e: \mathbb{E} \rightarrow \mathbb{E}$ defined by $e(\alpha)=N(\alpha)$ is the required function. Let $\alpha=a+A$ be an arbitrary external number. Then

$$
\alpha+e(\alpha)=\alpha+N(\alpha)=(a+A)+A=a+A=\alpha .
$$

Hence, for all $f: \mathbb{E} \rightarrow \mathbb{E}$ such that $\alpha+f(\alpha)=\alpha$

$$
e(\alpha)+f(\alpha)=A+f(\alpha)=\alpha-\alpha+f(\alpha)=-\alpha+\alpha=e(\alpha) .
$$

Proposition 4.4 There is a unique function $u: \mathbb{E} \backslash \mathcal{N} \rightarrow \mathbb{E}$ such that (i) $\alpha u(\alpha)=\alpha$ for all $\alpha \in \mathbb{E} \backslash \mathcal{N}$ and (ii) if $v: \mathbb{E} \backslash \mathcal{N} \rightarrow \mathbb{E}$ verifies $\alpha v(\alpha)=\alpha$ for all $\alpha \in \mathbb{E} \backslash \mathcal{N}$, then $u(\alpha) v(\alpha)=u(\alpha)$. In fact, $u(\alpha)=1+\frac{N(\alpha)}{\alpha}$.

Proof We prove that $u: \mathbb{E} \backslash \mathcal{N} \rightarrow \mathbb{E}$ defined by $u(\alpha)=1+\frac{N(\alpha)}{\alpha}$ is the required function. Let $\alpha=a+A$ be an arbitrary zeroless external number. Then using Proposition 3.3 and Lemma 3.5,

$$
\begin{aligned}
\alpha u(\alpha) & =\alpha\left(1+\frac{N(\alpha)}{\alpha}\right)=(a+A)\left(1+\frac{A}{a}\right) \\
& =a+\max \left(a \frac{A}{a}, A\right)=a+A=\alpha .
\end{aligned}
$$


Let $v: \mathbb{E} \backslash \mathcal{N} \rightarrow \mathbb{E}$ be such that $\alpha v(\alpha)=\alpha$ for all $\alpha \in \mathbb{E} \backslash \mathcal{N}$. Then applying Lemma 3.6

$$
\begin{aligned}
u(\alpha) v(\alpha) & =\left(1+\frac{N(\alpha)}{\alpha}\right) v(\alpha)=\left(1+\frac{A}{a}\right) v(\alpha) \\
& =\frac{(a+A) v(\alpha)}{a}=\frac{a+A}{a}=1+\frac{A}{a}=u(\alpha)
\end{aligned}
$$

Corollary 4.5 Let $\alpha$ be a zeroless external number. Then $u(\alpha) \neq e(\alpha)$.

Proposition 4.6 There is a unique function $s: \mathbb{E} \rightarrow \mathbb{E}$ such that $\alpha+s(\alpha)=e(\alpha)$ and $e(s(\alpha))=e(\alpha)$ for all $\alpha \in \mathbb{E}$. In fact, $s(\alpha)=-\alpha$.

Proof Let $\alpha=a+A$ be an arbitrary external number. We prove that $s: \mathbb{E} \rightarrow \mathbb{E}$ defined by $s(\alpha)=-\alpha$ is the required function. We have

$$
\alpha+s(\alpha)=(a+A)+(-a+A)=A=e(\alpha)
$$

and

$$
e(s(\alpha))=e(-a+A)=A=e(\alpha) .
$$

Suppose that $t: \mathbb{E} \rightarrow \mathbb{E}$ is such that $\alpha+t(\alpha)=e(\alpha)$ and $e(t(\alpha))=e(\alpha)$. Then $e(t(\alpha))=e(\alpha)=e(s(\alpha))$ and

$$
\begin{aligned}
t(\alpha) & =t(\alpha)+e(t(\alpha))=t(\alpha)+e(\alpha)=t(\alpha)+\alpha+s(\alpha) \\
& =e(\alpha)+s(\alpha)=e(s(\alpha))+s(\alpha)=s(\alpha) .
\end{aligned}
$$

Proposition 4.7 There is a unique function $d: \mathbb{E} \backslash \mathcal{N} \rightarrow \mathbb{E}$ such that $\alpha d(\alpha)=u(\alpha)$ and $u(d(\alpha))=u(\alpha)$, for all $\alpha \in \mathbb{E} \backslash \mathcal{N}$. In fact $d(\alpha)=\frac{1}{\alpha}=\frac{1}{a}+\frac{A}{a^{2}}$.

Proof Let $\alpha=a+A$ be an arbitrary external number. We prove that $d: \mathbb{E} \backslash \mathcal{N} \rightarrow \mathbb{E}$, defined by

$$
d(\alpha)=\frac{1}{\alpha}=\frac{1}{a}\left(\frac{1}{1+\frac{A}{a}}\right)=\frac{1}{a}\left(1+\frac{A}{a}\right)=\frac{1}{a}+\frac{A}{a^{2}}
$$

verifies the required conditions. We have

$$
\alpha d(\alpha)=(a+A)\left(\frac{1}{a}+\frac{A}{a^{2}}\right)=1+\frac{A}{a}=u(\alpha)
$$


and, by Proposition 4.4 and Proposition 3.3,

$$
u(d(\alpha))=u\left(\frac{1}{a}+\frac{A}{a^{2}}\right)=1+\frac{\frac{A}{a^{2}}}{\frac{1}{a}}=1+\frac{A}{a}=u(\alpha) .
$$

Uniqueness is shown in the same way as in the proof of Proposition 4.6.

It has been proved in Proposition 3.1 that always $e(\alpha+\beta)=e(\alpha)$ or $e(\alpha+\beta)=e(\beta)$. We prove an analogous property for multiplication.

Proposition 4.8 Let $\alpha$ and $\beta$ be zeroless external numbers. One has $u(\alpha \beta)=u(\alpha)$ or $u(\alpha \beta)=u(\beta)$.

Proof Let $\alpha=a+A$ and $\beta=b+B$ be zeroless. Then

$$
\begin{aligned}
u(\alpha \beta) & =u(a b+a B+b A)=1+\frac{a B+b A}{a b} \\
& =1+\max \left(\frac{B}{b}, \frac{A}{a}\right)=1+\max \left(\frac{N(\beta)}{\beta}, \frac{N(\alpha)}{\alpha}\right) .
\end{aligned}
$$

Hence, $u(\alpha \beta)=u(\alpha)$ or $u(\alpha \beta)=u(\beta)$.

The fact that addition and multiplication have the above properties in common suggests the definition of the following algebraic structure.

Definition 4.9 Given a set $G$ and a binary operation $*$ on $G$, we say that $(G, *)$ is an assembly if $G$ satisfies the following conditions:

(1) $\forall x \forall y \forall z(x *(y * z)=(x * y) * z)$;

(2) $\forall x \forall y(x * y=y * x)$;

(3) $\forall x \exists e(x * e=x \wedge \forall f(x * f=x \Longrightarrow e * f=e))$;

(4) $\forall x \exists s(x * s=e(x) \wedge e(s)=e(x))$;

(5) $\forall x \forall y(e(x * y)=e(x) \vee e(x * y)=e(y))$.

The functional notation used in Definition 4.9.4 and 4.9.5 is justified by the fact that the element $e$ of Definition 4.9.3 is unique. Indeed, if $e^{\prime}$ satisfies Definition 4.9.3, we have $e^{\prime}=e^{\prime} * e=e * e l=e$. Also $s$ is unique and may be considered functional. Indeed, if $s^{\prime}$ satisfies Definition 4.9.4 we have $s^{\prime}=s^{\prime} * e\left(s^{\prime}\right)=s^{\prime} * e(x)=s^{\prime} * x * s=x * s^{\prime} * s=$ $e(x) * s=e(s) * s=s$.

With the use of Propositions 4.3 - 4.8 one shows the following theorem. 
Theorem 4.10 The commutative regular semigroups $(\mathbb{E},+)$ and $(\mathbb{E} \backslash \mathcal{N},$.$) are assem-$ blies.

Classical examples of assemblies are $(A,+)$ with $A=\{k+n ! \mathbb{Z} \mid k, n \in \mathbb{Z}\}$, and $(B, \cup)$ where $B$ is the set of all ordinals less than a given ordinal.

Within assemblies the cancellation law takes the following form.

Theorem 4.11 (Cancellation law) Let $G$ be an assembly and let $x, y, z \in G$ be arbitrary. Then $x * y=x * z \Leftrightarrow e(x) * y=e(x) * z$.

Proof Suppose first that $x * y=x * z$. Then

$$
\begin{aligned}
x * y & =x * z \Longrightarrow s(x) * x * y=s(x) * x * z \\
& \Longrightarrow \quad e(x) * y=e(x) * z .
\end{aligned}
$$

Suppose now that $e(x) * y=e(x) * z$. Then

$$
\begin{aligned}
e(x) * y & =e(x) * z \Longrightarrow x * e(x) * y=x * e(x) * z \\
& \Longrightarrow \quad x * y=x * z .
\end{aligned}
$$

Hence $x * y=x * z \Leftrightarrow e(x) * y=e(x) * z$.

The neutral function $e$ and the inverse function $s$ have some natural properties, as stated in the next theorem.

Theorem 4.12 Let $(G, *)$ be an assembly. Then for all $x$ and $y \in G$

(1) $e(x) * e(x)=e(x)$.

(2) $e(x * y)=e(x) * e(y)$.

(3) $e(e(x))=e(x)$.

(4) $s(s(x))=x$.

(5) $s(x * y)=s(x) * s(y)$.

(6) $e(s(x))=s(e(x))=e(x)$.

(7) If $x \neq e(x)$, then $x \neq e(y)$.

Proof Let $x$ and $y$ be arbitrary elements of $G$.

(1) The equality follows directly from Definition 4.9 .3 with $f=e$. 
(2) By Definition 4.9.2 and 4.9.3 we have

$$
x * y * e(x) * e(y)=x * e(x) * y * e(y)=x * y .
$$

Then by Theorem 4.11,

$$
e(x * y) * e(x) * e(y)=e(x * y) .
$$

By Definition 4.9.5 we have that $e(x * y)=e(x)$ or $e(x * y)=e(y)$. Suppose that $e(x * y)=e(x)$. Then by formula (2) and Part 1 ,

$$
e(x * y)=e(x) * e(x) * e(y)=e(x) * e(y) .
$$

If $e(x * y)=e(y)$ the proof is analogous. Hence $e(x * y)=e(x) * e(y)$.

(3) By Definition 4.9.4, Part 1 and Part 2

$$
e(e(x))=e(x * s(x))=e(x) * e(s(x))=e(x) * e(x)=e(x) .
$$

(4) Observe that by Definition 4.9.4

$$
e(s(s(x)))=e(s(x))=e(x)=s(x) * x .
$$

Hence

$$
s(s(x))=s(s(x)) * e(s(s(x)))=s(s(x)) * s(x) * x=e(s(x)) * x=e(x) * x=x .
$$

(5) By Part 2 and Definition 4.9.4

$s(x * y) * x * y=e(x * y)=e(x) * e(y)=s(x) * x * s(y) * y=s(x) * s(y) * x * y$.

Then by Theorem 4.11

$$
s(x * y) * e(x * y)=s(x) * s(y) * e(x * y) .
$$

Again using Definition 4.9.4 we obtain

$$
\begin{aligned}
s(x * y) * e(s(x * y)) & =s(x) * s(y) * e(s(x)) * e(s(y)) \\
& =s(x) * e(s(x)) * s(y) * e(s(y)),
\end{aligned}
$$

and consequently

$$
s(x * y)=s(x) * s(y) .
$$

(6) By Definition 4.9.4 we only have to show that $s(e(x))=e(x)$. Using Part 4 and Part 5 we derive

$$
e(x)=s(x) * x=s(x) * s(s(x))=s(x * s(x))=s(e(x)) .
$$

(7) Suppose $y \in G$ is such that $x=e(y)$. Then, using Part 3,

$$
e(x)=e(x * e(x))=e(e(y) * e(x))=e(e(y) * e(e(y)))=e(e(y))=e(y)=x .
$$

This proves Part 7. 
Theorem 4.11 and 4.12 have the following consequences for the addition and multiplication of external numbers.

Corollary 4.13 Let $\alpha, \beta, \gamma$ be external numbers. Then

(1) $\alpha+\beta=\alpha+\gamma \Leftrightarrow e(\alpha)+\beta=e(\alpha)+\gamma$.

(2) The function $e$ is idempotent for sum and for composition.

(3) The function $e$ is an homomorphism for addition.

(4) The composition of $s$ with itself is the identity map.

(5) The function $s$ is an homomorphism for addition.

(6) $s(e(\alpha))=e(\alpha)$.

(7) If $\alpha \neq e(\alpha)$, then $\alpha \neq e(\beta)$.

Corollary 4.14 Let $\alpha, \beta, \gamma$ be zeroless external numbers. Then

(1) $\alpha \beta=\alpha \gamma \Leftrightarrow u(\alpha) \beta=u(\alpha) \gamma$.

(2) The function $u$ is idempotent for multiplication and for composition.

(3) The function $u$ is an homomorphism for multiplication.

(4) The composition of $d$ with itself is the identity map.

(5) The function $d$ is an homomorphism for multiplication.

(6) $d(u(\alpha))=u(\alpha)$.

(7) If $\alpha \neq u(\alpha)$, then $\alpha \neq u(\beta)$.

We finish by exploring the connection between the neutral and inverse functions of addition and multiplication.

Proposition 4.15 The functions $e$ and $s$ have the following properties with respect to multiplication. For all $\alpha, \beta \in \mathbb{E}$

(1) $e(\alpha \beta)=\beta e(\alpha)+\alpha e(\beta)$.

(2) $s(\alpha \beta)=s(\alpha) \beta=\alpha s(\beta)$.

(3) $\alpha \beta=e(\alpha \beta) \Leftrightarrow \alpha=e(\alpha) \vee \beta=e(\beta)$. 
Proof Let $\alpha=a+A$ and $\beta=b+B$ be arbitrary external numbers. By Proposition 4.3 we have $e(\alpha)=N(\alpha)=A$ and $e(\beta)=N(\beta)=B$.

(1) We have

$$
\begin{aligned}
e(\alpha \beta) & =e((a+A)(b+B)) \\
& =e(a b+a B+b A+A B)=a B+b A+A B
\end{aligned}
$$

and

$$
\begin{aligned}
\beta e(\alpha)+\alpha e(\beta) & =(b+B) A+(a+A) B \\
& =b A+A B+a B+A B=a B+b A+A B .
\end{aligned}
$$

Therefore $e(\alpha \beta)=\beta e(\alpha)+\alpha e(\beta)$.

(2) It holds that

$$
\begin{aligned}
s(\alpha \beta) & =s(a b+a B+b A+A B) \\
& =-a b+a B+b A+A B \\
& =(-a+A)(b+B)=s(\alpha) \beta .
\end{aligned}
$$

(3) To prove the direct implication we assume that $\alpha \beta=e(\alpha \beta)$. Then

$$
a b+a B+b A+A B=a B+b A+A B .
$$

This implies that $a b \in a B, a b \in b A$ or $a b \in A B$. Suppose that $\alpha \neq e(\alpha)$ and $\beta \neq e(\beta)$. Then $a \notin A$ and $b \notin B$. Hence $a b \notin a B, a b \notin b A$ and $a b \notin A B$, a contradiction. We conclude that

$$
\alpha \beta=e(\alpha \beta) \Rightarrow \alpha=e(\alpha) \vee \beta=e(\beta) .
$$

Assume now that $\alpha=e(\alpha)$ or $\beta=e(\beta)$. If $\alpha=e(\alpha)$, by Lemma 3.4

$$
\alpha \beta=A \beta=A b+A B=e(\alpha \beta) .
$$

The other case is analogous. Hence

$$
\alpha=e(\alpha) \vee \beta=e(\beta) \Rightarrow \alpha \beta=e(\alpha \beta) .
$$

Combining (3) and (4), we obtain Part 3.

Observe that the interpretation of neutrices as generalized zeros is further justified by Part 3 of the previous proposition, which states that in a sense zero divisors can only be neutrices.

This interpretation is enhanced by the next proposition, stating that neutral elements for addition are invariant for neutral elements for multiplication. 
Proposition 4.16 For all $\alpha \in \mathbb{E}$ and $\beta \in \mathbb{E} \backslash \mathcal{N}$ it holds that $e(\alpha) u(\beta)=e(\alpha)$.

Proof Let $\alpha=a+A$ be an external number and assume that $\beta=b+B$ is zeroless. Then by Proposition 3.3

$$
e(\alpha) u(\beta)=A\left(1+\frac{B}{b}\right)=A+\frac{A B}{b}=A=e(\alpha) .
$$

The final proposition determines the neutral function for addition of the neutral function for multiplication.

Proposition 4.17 For all $\alpha \in \mathbb{E} \backslash \mathcal{N}$ it holds that $e(u(\alpha))=e(\alpha) d(\alpha)$.

Proof Assume that $\alpha=a+A$ is zeroless. Then by Proposition 4.7 and 3.3

$$
e(\alpha) d(\alpha)=A\left(\frac{1}{a}+\frac{A}{a^{2}}\right)=\frac{A}{a}=e(u(\alpha)) .
$$

\section{Distributivity}

External numbers are intervals of real numbers and therefore multiplication is $s u b$ distributive with respect to addition [18], i.e. for all external numbers $\alpha, \beta$ and $\gamma$

$$
\alpha(\beta+\gamma) \subseteq \alpha \beta+\alpha \gamma .
$$

However, proper distributivity does not always hold. Take for example $\alpha=\oslash, \beta=\omega+1$ and $\gamma=-\omega$, where $\omega$ is an unlimited number. Then $\alpha(\beta+\gamma)=\oslash((\omega+1)-\omega)=\oslash$ and $\alpha \beta+\alpha \gamma=(\omega+1) \oslash-\omega \oslash=\omega \oslash$. Nevertheless the validity of the distributive law can be completely characterized. To this end we introduce the following notions.

Definition 5.1 Let $\alpha \in \mathbb{E}$. If $\alpha$ is zeroless, we define the relative uncertainty of $\alpha$ to be the neutrix $\mathcal{R}(\alpha)=\frac{A}{a}$. If $\alpha \in \mathcal{N}$, we define $\mathcal{R}(\alpha)=\mathbb{R}$.

Remark 5.2 Let $\alpha=a+A$ be a zeroless external number. Then $\mathcal{R}(\alpha) \subseteq \oslash$ by Proposition 3.3. Moreover $\alpha=a(1+\mathcal{R}(\alpha))$, because Lemma 3.6 implies that $a(1+\mathcal{R}(\alpha))=a+a \mathcal{R}(\alpha)=a+A=\alpha$. 
Definition 5.3 Let $\alpha$ and $\beta$ be external numbers. We say that $\alpha$ is (asymptotically) more precise than $\beta$ if $\mathcal{R}(\alpha) \subseteq \mathcal{R}(\beta)$.

Observe that each zeroless external number is more precise than any neutrix.

Definition 5.4 Let $A$ be a neutrix and $\beta$ and $\gamma$ be external numbers. Then $\beta$ and $\gamma$ are called opposite with respect to $A$ if $(\beta+\gamma) A \subset \max (|\beta|,|\gamma|) A$.

Examples. Two real numbers $b$ and $c$ which are opposite, i.e. such that $b=-c$ are opposite with respect to all neutrices $N \supset\{0\}$. Appreciable real numbers $\beta$ and $\gamma$ are opposite with respect to $\oslash$ and $£$ if and only if $\beta \simeq-\gamma$. Let $\omega$ be unlimited. Then $\omega+1$ and $-\omega$ are opposite with respect to $\oslash$ without being nearly equal, because as we already saw

$$
((\omega+1)-\omega) \oslash=\oslash \subset \omega \oslash=(\omega+1) \oslash .
$$

If two numbers are opposite with respect to a given neutrix, none of them can be a neutrix. To see this observe first that $\beta+\gamma=\max (|\beta|,|\gamma|)$ if both are a neutrix or if one of them, say $\beta$, is a neutrix and $\gamma \subseteq \beta$. In the remaining case we may suppose that $\beta$ is a neutrix and $\beta<|\gamma|$. Clearly $\gamma A \subseteq(\beta+\gamma) A$. Since $\beta / \gamma \subseteq \oslash$, it follows from Lemma 3.6 that $\beta+\gamma \subseteq \oslash \gamma+\gamma=(1+\oslash) \gamma$, so $(\beta+\gamma) A \subseteq(1+\oslash) \gamma A=\gamma A$. Hence $(\beta+\gamma) A=\gamma A=\max (|\beta|,|\gamma|) A$.

Numbers of the same sign are never opposite with respect to a given neutrix. In fact, two external numbers $\beta$ and $\gamma$ which are opposite with respect to a given neutrix $A$ must satisfy $\beta / \gamma \subseteq-1+\oslash$. Indeed, if $-1+a \in \beta / \gamma$ with $a \nsucceq 0$,

$$
(\beta+\gamma) A= \begin{cases}\beta A=\max (|\beta|,|\gamma|) A & |a| \simeq \infty \\ (-1+a) \gamma A=\gamma A=\beta A & -1+a \text { appreciable } \\ \gamma A=\max (|\beta|,|\gamma|) A & -1+a \simeq 0 .\end{cases}
$$

The latter observation enables a characterization in terms of absorbers: If $\beta$ and $\gamma$ are opposite with respect to $A$, both $(\beta+\gamma) A \subset \beta A$ and $(\beta+\gamma) A \subset \gamma A$, hence $(\beta+\gamma) / \gamma$ and $(\beta+\gamma) / \beta$ are absorbers of $A$. Oppositeness is directly related to distributivity. Indeed, if $\beta$ and $\gamma$ are opposite with respect to $A$,

$$
(\beta+\gamma) A \subset \max (|\beta|,|\gamma|) A=\max (\beta A, \gamma A)=\beta A+\gamma A,
$$

and if $\beta$ and $\gamma$ are not opposite with respect to $A$,

$$
(\beta+\gamma) A=\max (|\beta|,|\gamma|) A=\max (\beta A, \gamma A)=\beta A+\gamma A .
$$

The next two lemmas are useful in dealing with oppositeness with respect to linear combinations of neutrices. 
Lemma 5.5 Let $\alpha=a+A, \beta=b+B$ and $\gamma=c+C$ be external numbers. Let $M$ and $N$ be neutrices.

(1) If $\alpha$ and $\beta$ are not opposite with respect to $M$ nor $N$, then $\alpha$ and $\beta$ are not opposite with respect to $M+N$.

(2) If $\alpha$ and $\beta$ are not opposite with respect to $M$, then $\alpha$ and $\beta$ are not opposite with respect to $\gamma M$.

Proof Suppose that $\alpha$ and $\beta$ are not opposite with respect to $M$ nor $N$.

(1) As a consequence of Proposition 3.1 and formula (5) one has

$$
\begin{aligned}
(\alpha+\beta)(M+N) & =(\alpha+\beta) M+(\alpha+\beta) N \\
& =\alpha M+\beta M+\alpha N+\beta N \\
& =\alpha(M+N)+\beta(M+N) .
\end{aligned}
$$

(2) By formula (5)

$$
\begin{aligned}
(\alpha+\beta) \gamma M & =\gamma(\alpha+\beta) M \\
& =\gamma(\alpha M+\beta M) \\
& =\alpha \gamma M+\beta \gamma M
\end{aligned}
$$

We are now able to state the criterion for distributivity:

Theorem 5.6 Let $\alpha, \beta$ and $\gamma$ be external numbers. Then $\alpha(\beta+\gamma)=\alpha \beta+\alpha \gamma$ if and only if (i) $\alpha$ is more precise than $\beta$ or $\gamma$, or (ii) $\beta$ and $\gamma$ are not opposite with respect to $N(\alpha)$.

If one of the numbers is a neutrix, we may identify the following special cases:

Theorem 5.7 Let $\alpha, \beta$ and $\gamma$ be external numbers.

(1) If $\alpha \in \mathcal{N}$ and neither $\beta \in \mathcal{N}$, nor $\gamma \in \mathcal{N}$, then $\alpha(\beta+\gamma)=\alpha \beta+\alpha \gamma$ if and only if $\beta$ and $\gamma$ are not opposite with respect to $\alpha$.

(2) If $\beta \in \mathcal{N}$, or $\gamma \in \mathcal{N}$, then $\alpha(\beta+\gamma)=\alpha \beta+\alpha \gamma$.

A second important special case concerns external numbers of the same sign. 
Theorem 5.8 If $\alpha$ is an external number and $\beta$ and $\gamma$ are external numbers of the same sign, then $\alpha(\beta+\gamma)=\alpha \beta+\alpha \gamma$.

Let us illustrate the above results with some examples:

(1) Let $\alpha=1+\oslash, \beta=1+\varepsilon$ with $\varepsilon \simeq 0$, and $\gamma=1$. Then $\alpha(\beta+\gamma)=\alpha \beta+\alpha \gamma$ by Theorem 5.8. The equality follows also from Theorem 5.6 (ii). On one hand,

$$
\begin{aligned}
\alpha(\beta+\gamma) & =(1+\oslash)(1+\varepsilon+1)=(1+\oslash)(2+\varepsilon) \\
& =2+\varepsilon+(2+\varepsilon) \oslash \quad \text { by Theorem 5.6 (ii) } \\
& =2+\varepsilon+\oslash \quad \text { because } 2+\varepsilon \text { is appreciable } \\
& =2+\oslash .
\end{aligned}
$$

On the other hand,

$$
\begin{aligned}
\alpha \beta+\alpha \gamma & =(1+\oslash)(1+\varepsilon)+(1+\oslash) 1 \\
& =1+\oslash+(1+\oslash) \varepsilon+1+\oslash \quad \text { by Theorem 5.6 (ii) } \\
& =1+\oslash+\varepsilon+\varepsilon \oslash+1+\oslash \quad \text { by Theorem 5.6 (ii) } \\
& =2+\oslash .
\end{aligned}
$$

(2) Let $\alpha=1+\oslash, \beta=1+\varepsilon$ with $\varepsilon \simeq 0$, and $\gamma=-1$. Then

$$
\alpha(\beta+\gamma)=(1+\oslash)(1+\varepsilon-1)=(1+\oslash) \varepsilon=\varepsilon+\varepsilon \oslash
$$

and

$$
\begin{aligned}
\alpha \beta+\alpha \gamma & =(1+\oslash)(1+\varepsilon)+(1+\oslash)(-1) \\
& =1+\oslash+(1+\oslash) \varepsilon-1+\oslash \quad \text { by Theorem } 5.6 \text { (ii) } \\
& =\oslash+\varepsilon+\oslash \varepsilon \quad \text { by Theorem } 5.6 \text { (ii) } \\
& =\oslash .
\end{aligned}
$$

Because $\varepsilon \oslash \subset \oslash$, subdistributivity holds, but distributivity does not. This is in line with the fact that $\alpha$ is less precise than both $\beta$ and $\gamma$ and $1+\varepsilon$ and -1 are opposite with respect to $\oslash$. If we change $\beta$ into $\beta^{\prime}=1+\oslash$, then $\alpha$ is as precise as $\beta^{\prime}$ and one verifies indeed that

$$
\alpha\left(\beta^{\prime}+\gamma\right)=\oslash=\alpha \beta^{\prime}+\alpha \gamma .
$$

If $\varepsilon$ is appreciable, the numbers $1+\varepsilon$ and -1 are no longer opposite with respect to $\oslash$ and we see that

$$
\alpha(\beta+\gamma)=\varepsilon+\varepsilon \oslash=\varepsilon+\oslash=\oslash+\varepsilon+\oslash \varepsilon=\alpha \beta+\alpha \gamma .
$$


(3) If $\alpha=\oslash, \beta=\omega+£, \gamma=-\omega+\oslash$, where $\omega \simeq+\infty$, distributivity does not hold by Theorem 5.7.1, for $\beta$ and $\gamma$ are opposite with respect to $\oslash$. Indeed, one shows with the aid of Theorem 5.7.2 that

$$
\begin{aligned}
\alpha(\beta+\gamma) & =\oslash £=\oslash \subset \oslash \omega \\
& =\oslash \omega+\oslash £=\oslash(\omega+£)=\alpha \max (|\beta|,|\gamma|) .
\end{aligned}
$$

(4) If $\alpha=\sqrt{\omega}+£, \beta=\omega+£, \gamma=£$, where $\omega \simeq+\infty$, distributivity holds. Indeed, by Theorem 5.6 (i) and Theorem 5.7.2

$$
\begin{aligned}
\alpha(\beta+\gamma) & =(\sqrt{\omega}+£)(\omega+£)=(\sqrt{\omega}+£) \omega+(\sqrt{\omega}+£) £ \\
& =\omega \sqrt{\omega}+£ \omega+\sqrt{\omega} £+£ £=\omega \sqrt{\omega}+£ \omega .
\end{aligned}
$$

Note that we have calculated in fact $\alpha \beta$, and that $\alpha \gamma=(\sqrt{\omega}+£) £=$ $\sqrt{\omega} £+£ £=\sqrt{\omega} £$ is contained in $N(\alpha \beta)$. Hence $\alpha \beta+\alpha \gamma=\alpha \beta=\alpha(\beta+\gamma)$.

Some of these calculations may be obtained directly by the definition of multiplication for external numbers. In fact this rule plays a substantial part in the proof of Theorem 5.6. We prove Theorem 5.6 considering two separate cases: the case where one of the numbers is a neutrix (Section 5.1) and the case where none of the external numbers is a neutrix (Section 5.2).

\subsection{Distributivity with neutrices}

In this section we prove Theorem 5.6 in the case where at least one of the external numbers $\alpha, \beta, \gamma$ is a neutrix. We have to consider two subcases, (i) $\beta$ or $\gamma$ is a neutrix and (ii) $\alpha$ is a neutrix.

Proof of Theorem 5.6 in the case where $\beta$ or $\gamma$ is a neutrix: We have $\mathcal{R}(\beta)=\mathbb{R}$ or $\mathcal{R}(\gamma)=\mathbb{R}$. Then the criterion

$$
\mathcal{R}(\alpha) \leq \max (\mathcal{R}(\beta), \mathcal{R}(\gamma)) \vee(\beta+\gamma) A=A \max (|\beta|,|\gamma|)
$$

is trivially satisfied. Conversely, suppose without loss of generality that $\gamma=C \in \mathcal{N}$. Because $a B, a C, b A, A B, A C$ are neutrices, by Proposition 3.1

$$
\begin{aligned}
\alpha(\beta+\gamma) & =(a+A)(b+\max (B, C)) \\
& =a b+a \max (B, C)+b A+A \max (B, C) \\
& =a b+\max (a B, a C, b A, A B, A C)
\end{aligned}
$$


and

$$
\begin{aligned}
\alpha \beta+\alpha \gamma & =a b+\max (a B, b A, A B)+\max (a C, A C) \\
& =a b+\max (a B, a C, b A, A B, A C) .
\end{aligned}
$$

Hence $\alpha(\beta+\gamma)=\alpha \beta+\alpha \gamma$

For the sake of clarity we mention in the form of a corollary the special cases which involve at least two neutrices.

Corollary 5.9 Let $\alpha$ be an arbitrary external number and let $B, C \in \mathcal{N}$. Then $\alpha(B+C)=\alpha B+\alpha C$.

Corollary 5.10 If $A, B, C \in \mathcal{N}$, then $A(B+C)=A B+A C$.

Corollary 5.11 Let $A, C \in \mathcal{N}$ and let $\beta$ be zeroless. Then $A(\beta+C)=A \beta+A C$.

Proof of Theorem 5.6 in the case where $\alpha$ is a neutrix: Let $\alpha=A$ be an arbitrary neutrix. Without loss of generality we may assume that $|\beta| \geq|\gamma|$. Firstly suppose that $A(\beta+\gamma)=A \beta+A \gamma$. Then $A \beta+A \gamma=A \beta=A \max (|\beta|,|\gamma|)$. Hence $\beta$ and $\gamma$ are not opposite with respect to $A$.

Suppose now that $A$ is more precise than $\beta$ or $\gamma$, or that $\beta$ and $\gamma$ are not opposite with respect to $A$. In the first case $\beta$ or $\gamma$ has to be a neutrix because $\mathcal{R}(A)=\mathbb{R}$. This case is contained in Corollaries 5.10 and 5.11. In the second case $A(\beta+\gamma)=A \max (|\beta|,|\gamma|)=A \beta=A \beta+A \gamma$.

\subsection{Distributivity with zeroless external numbers}

Let $\alpha, \beta$ and $\gamma$ be zeroless external numbers. We always write $\alpha=a+A, \beta=b+B$ and $\gamma=c+C$, where $a, b$ and $c$ are real numbers and $A, B$ and $C$ are neutrices, with $A<|a|, B<|b|$ and $C<|c|$. Hence by Remark $5.2 \alpha=a(1+\mathcal{R}(\alpha))$, $\beta=b(1+\mathcal{R}(\beta))$ and $\gamma=c(1+\mathcal{R}(\gamma))$.

In order to prove the criterion for distributivity we suppose without loss of generality that $|\beta| \geq|\gamma|$. Then we may also suppose that $0<\left|\frac{c}{b}\right| \leq 1$.

We prove the criterion for distributivity first in the case that $a=1, b=1$ and $0<|c| \leq 1$; then $A \leq \oslash$ and $B \leq \oslash$ by Lemma 3.3. The general case will be obtained by rescaling.

To do so, we need to give direct proofs of distributivity in some relatively easy special cases. 
Lemma 5.12 One has $a(\beta+\gamma)=a \beta+a \gamma$.

Proof By Lemma 3.6 and Corollary 5.9 we have

$$
\begin{aligned}
a(\beta+\gamma) & =a((b+c)+\max (B, C)) \\
& =a(b+c)+a \max (B, C) \\
& =a b+a c+a B+a C \\
& =a(b+B)+a(c+C) \\
& =a \beta+a \gamma .
\end{aligned}
$$

Proposition 5.13 If $\alpha, \beta$ and $\gamma$ are external numbers with $\beta$ and $\gamma$ not opposite with respect to $£$, then $\alpha(\beta+\gamma)=\alpha \beta+\alpha \gamma$.

Proof We assume without loss of generality that $\alpha$ and $\beta$ are positive. Because $A £=A$, by Lemma 5.5.2 and by formula (5) it holds that $(\beta+\gamma) A=\beta A+\gamma A$. Notice that $\beta+\gamma$ is zeroless. If not, both $£(\beta+\gamma)=£(B+C)=B+C$ and $£(\beta+\gamma)=£ \beta \geq b$, with $b>B$ and $b \geq|c|>C$, a contradiction. Then by Lemma 3.6 and Lemma 5.12

$$
\begin{aligned}
\alpha(\beta+\gamma) & =a(\beta+\gamma)+A(\beta+\gamma) \\
& =a \beta+a \gamma+A \beta+A \gamma \\
& =\alpha \beta+\alpha \gamma
\end{aligned}
$$

Lemma 5.14 Assume that $a=1, b=1$ and $0<|c| \leq 1$. Then

(1) If $\beta+\gamma \in \mathcal{N}$,

$$
\alpha(\beta+\gamma)=\alpha \beta+\alpha \gamma \Leftrightarrow A \leq \max (B, C) \Leftrightarrow \alpha \beta=\beta \vee \alpha \gamma=\gamma .
$$

(2) If $\beta+\gamma \notin \mathcal{N}$,

$$
\begin{aligned}
\alpha(\beta+\gamma) & =\alpha \beta+\alpha \gamma \Leftrightarrow A \leq \max (B, C) \vee(1+c) A=A \\
& \Leftrightarrow \alpha \beta=\beta \vee \alpha \gamma=\gamma \vee(\beta+\gamma) A=A
\end{aligned}
$$

Proof First observe that, by Lemma 3.5 and Lemma 3.4, we have

$$
\alpha \beta=(1+A)(1+B)=1+A+B
$$


and

$$
\alpha \gamma=(1+A)(c+C)=c+c A+C .
$$

Hence

(8) $\alpha \beta+\alpha \gamma=1+A+B+c+c A+C=1+c+A+B+C=1+c+\max (A, B, C)$.

1. We start with the first equivalence. By hypothesis and Lemma 3.6, we have

$$
\alpha(\beta+\gamma)=(1+A)(B+C)=\max (B, C) .
$$

Hence, by formula (8) and formula (9), $\alpha(\beta+\gamma)=\alpha \beta+\alpha \gamma$ if and only if $A \leq$ $\max (B, C)$.

To prove the second equivalence, suppose first that $\alpha \beta=\beta$ or $\alpha \gamma=\gamma$. In the first case, by formula (6)

$$
1+A+B=1+B
$$

which implies that $A \leq B$. In the second case by formula (7)

$$
c+c A+C=c+C .
$$

Because $\beta$ and $\gamma$ are zeroless, one has $B, C \subseteq \oslash$. Now $\beta+\gamma=1+c+B+C \subseteq$ $1+c+\oslash \in \mathcal{N}$, which implies that $c \simeq-1$, hence $c A=A$. Then we derive from (10) that $c+A+C=c+C$, so $A \leq C$. We conclude that $A \leq \max (B, C)$. Conversely, suppose that $A \leq \max (B, C)$. Hence $A \leq B$ or $A \leq C$. Then clearly $1+A+B=1+B$ or $c+A+C=c+C$ and $\alpha \beta=\beta$ or $\alpha \gamma=\gamma$ by formula (6) and formula (7). Hence

$$
A \leq \max (B, C) \Leftrightarrow \alpha \beta=\beta \vee \alpha \gamma=\gamma .
$$

2. One has $\beta+\gamma \neq \max (B, C)$. Hence

$$
(\beta+\gamma) A=(1+B+c+C) A=(1+c) A .
$$

We prove first that

$$
\alpha(\beta+\gamma)=\alpha \beta+\alpha \gamma \Leftrightarrow A \leq \max (B, C) \vee(1+c) A=A .
$$

Lemma 3.5 yields

$$
\begin{aligned}
\alpha(\beta+\gamma) & =(1+A)(1+B+c+C) \\
& =1+c+B+C+(1+c) A .
\end{aligned}
$$

If $A \leq \max (B, C)$, because $(1+c) A \subseteq A$ both $\alpha \beta+\alpha \gamma=1+c+\max (B, C)$ and $\alpha(\beta+\gamma)=1+c+\max (B, C)$. If $(1+c) A=A$, we conclude from (8) that $\alpha \beta+\alpha \gamma=\alpha(\beta+\gamma)$. If $\alpha \beta+\alpha \gamma=\alpha(\beta+\gamma)$, then, by formula (8) it holds that 
$A+B+C=B+C+(1+c) A$, so $A \leq \max (B, C)$ or $A=(1+c) A$. Hence formula (12) holds. Finally we prove that

$$
A \leq \max (B, C) \vee(1+c) A=A \Leftrightarrow \alpha \beta=\beta \vee \alpha \gamma=\gamma \vee(\beta+\gamma) A=A .
$$

By (6) and (7) we have

$$
\alpha \beta=\beta \Leftrightarrow A \leq B
$$

and

$$
\alpha \gamma=\gamma \Leftrightarrow C+c A=C .
$$

Assume $A \leq \max (B, C)$ or $(1+c) A=A$. If $A \leq B$, then $\alpha \beta=\beta$. If $A \leq C$, then $C+c A=C$, hence $\alpha \gamma=\gamma$. If $(1+c) A=A$, then $(\beta+\gamma) A=(1+c+B+C) A=$ $(1+c) A+B A+C A=A$. Assume now that $\alpha \beta=\beta$ or $\alpha \gamma=\gamma$, or $(\beta+\gamma) A=A$. If $\alpha \beta=\beta$, then $A \leq B \leq \max (B, C)$. If $\alpha \gamma=\gamma$, then $c A \leq C$. If $c A=A$ we have that $A \leq C \leq \max (B, C)$. If $c A<A$ then $1+c \simeq 1$, hence $(1+c) A=A$. If $(\beta+\gamma) A=A$, then $(1+c) A=A$, because $\beta+\gamma$ is zeroless. We conclude that formula (13) holds.

We are now able to characterize distributivity for zeroless external numbers:

Theorem 5.15 (1) If $\beta+\gamma \in \mathcal{N}$,

$$
\alpha(\beta+\gamma)=\alpha \beta+\alpha \gamma \Leftrightarrow \mathcal{R}(\alpha) \leq \max (\mathcal{R}(\beta), \mathcal{R}(\gamma)) .
$$

(2) If $\beta+\gamma \notin \mathcal{N}$

$$
\begin{aligned}
& \alpha(\beta+\gamma)=\alpha \beta+\alpha \gamma \\
& \Leftrightarrow \mathcal{R}(\alpha) \leq \max (\mathcal{R}(\beta), \mathcal{R}(\gamma)) \vee(\beta+\gamma) A=A \max (|\beta|,|\gamma|) .
\end{aligned}
$$

Proof First, we put the products in a convenient form. Then by Lemma 3.4 and by Lemma 5.12 we have

$$
\begin{aligned}
\alpha(\beta+\gamma) & =a(1+\mathcal{R}(\alpha))(b(1+\mathcal{R}(\beta))+c(1+\mathcal{R}(\gamma))) \\
& =a b(1+\mathcal{R}(\alpha))\left(1+\mathcal{R}(\beta)+\frac{c}{b}+\frac{c}{b} \mathcal{R}(\gamma)\right)
\end{aligned}
$$

and

$$
\begin{aligned}
(16) \alpha \beta+\alpha \gamma & =a(1+\mathcal{R}(\alpha)) b(1+\mathcal{R}(\beta))+a(1+\mathcal{R}(\alpha)) c(1+\mathcal{R}(\gamma)) \\
& =a b\left((1+\mathcal{R}(\alpha))(1+\mathcal{R}(\beta))+(1+\mathcal{R}(\alpha))\left(\frac{c}{b}+\frac{c}{b} \mathcal{R}(\gamma)\right)\right)
\end{aligned}
$$


From (15) and (16) we conclude that distributivity is equivalent to

$$
\begin{aligned}
& (1+\mathcal{R}(\alpha))\left(1+\mathcal{R}(\beta)+\frac{c}{b}+\frac{c}{b} \mathcal{R}(\gamma)\right) \\
= & (1+\mathcal{R}(\alpha))(1+\mathcal{R}(\beta))+(1+\mathcal{R}(\alpha))\left(\frac{c}{b}+\frac{c}{b} \mathcal{R}(\gamma)\right) .
\end{aligned}
$$

Since by assumption $|\gamma| \leq|\beta|$ and $|c| \leq|b|$, we are able to apply Lemma 5.14. To prove Part 1, suppose that $\beta+\gamma \in \mathcal{N}$. Then $b+B+c+C=B+C \subseteq \oslash b+\oslash c=\oslash b$, so $1+\frac{c}{b}+\oslash \subseteq \oslash$. Hence $\frac{c}{b} \simeq-1$. This implies that $\frac{c}{b} \mathcal{R}(\gamma)=\mathcal{R}(\gamma)$. Then

$$
\mathcal{R}(\alpha) \leq \max \left(\mathcal{R}(\beta), \frac{c}{b} \mathcal{R}(\gamma)\right) \Leftrightarrow \mathcal{R}(\alpha) \leq \max (\mathcal{R}(\beta), \mathcal{R}(\gamma)) .
$$

Hence, by Lemma 5.14.1,

$$
\begin{aligned}
\alpha(\beta+\gamma) & =\alpha \beta+\alpha \gamma \Leftrightarrow \mathcal{R}(\alpha) \leq \max \left(\mathcal{R}(\beta), \frac{c}{b} \mathcal{R}(\gamma)\right) \\
& \Leftrightarrow \mathcal{R}(\alpha) \leq \max (\mathcal{R}(\beta), \mathcal{R}(\gamma)) .
\end{aligned}
$$

To prove Part 2, suppose that $\beta+\gamma \notin \mathcal{N}$. Then, by Lemma 5.14.2 and formula (17)

$$
\begin{aligned}
\alpha(\beta+\gamma)= & \alpha \beta+\alpha \gamma \Leftrightarrow(1+\mathcal{R}(\alpha))(1+\mathcal{R}(\beta))=1+\mathcal{R}(\beta) \\
& \vee(1+\mathcal{R}(\alpha))\left(\frac{c}{b}+\frac{c}{b} \mathcal{R}(\gamma)\right)=\frac{c}{b}+\frac{c}{b} \mathcal{R}(\gamma) \\
& \vee\left(1+\mathcal{R}(\beta)+\frac{c}{b}+\frac{c}{b} \mathcal{R}(\gamma)\right) A=A .
\end{aligned}
$$

First, we prove the direct implication of (14), using (18). With respect to (18) there are three cases to consider:

(i) $(1+\mathcal{R}(\alpha))(1+\mathcal{R}(\beta))=1+\mathcal{R}(\beta)$, (ii) $(1+\mathcal{R}(\alpha))\left(\frac{c}{b}+\frac{c}{b} \mathcal{R}(\gamma)\right)=\frac{c}{b}+\frac{c}{b} \mathcal{R}(\gamma)$ and (iii) $\left(1+\mathcal{R}(\beta)+\frac{c}{b}+\frac{c}{b} \mathcal{R}(\gamma)\right) A=A$.

(i) By Lemma 3.5

$$
1+\mathcal{R}(\alpha)+\mathcal{R}(\beta)=1+\mathcal{R}(\beta),
$$

hence

$$
\mathcal{R}(\alpha) \leq \mathcal{R}(\beta) .
$$

This implies that $\mathcal{R}(\alpha) \leq \max (\mathcal{R}(\beta), \mathcal{R}(\gamma))$.

(ii) One has similarly to (19)

implying that

$$
\frac{c}{b}(1+\mathcal{R}(\alpha)+\mathcal{R}(\gamma))=\frac{c}{b}(1+\mathcal{R}(\gamma)),
$$

$$
\mathcal{R}(\alpha) \leq \mathcal{R}(\gamma)
$$


Hence $\mathcal{R}(\alpha) \leq \max (\mathcal{R}(\beta), \mathcal{R}(\gamma))$.

(iii) By Lemma 3.6

$$
(b+B+c+C) A=b A,
$$

and therefore

$$
(\beta+\gamma) A=\beta A=A \max (|\beta|,|\gamma|) .
$$

Combining the three cases, we conclude that

(20) $\alpha(\beta+\gamma)=\alpha \beta+\alpha \gamma$

$$
\Rightarrow \mathcal{R}(\alpha) \leq \max (\mathcal{R}(\beta), \mathcal{R}(\gamma)) \vee(\beta+\gamma) A=A \max (|\beta|,|\gamma|) .
$$

To prove the reverse implication we need to consider two cases:

(i) $(\beta+\gamma) A=A \max (|\beta|,|\gamma|)$ and (ii) $\mathcal{R}(\alpha) \leq \max (\mathcal{R}(\beta), \mathcal{R}(\gamma))$.

(i) One has $(\beta+\gamma) A=|\beta| A$. Then

$$
(b+B+c+C) A=(b+B) A .
$$

This implies that

$$
\left(1+\mathcal{R}(\beta)+\frac{c}{b}+\frac{c}{b} \mathcal{R}(\gamma)\right) A=A .
$$

Then by (18) we conclude that

$$
(\beta+\gamma) A=A \max (|\beta|,|\gamma|) \Rightarrow \alpha(\beta+\gamma)=\alpha \beta+\alpha \gamma .
$$

(ii) If $\mathcal{R}(\alpha) \leq \max (\mathcal{R}(\beta), \mathcal{R}(\gamma))$, then $\mathcal{R}(\alpha) \leq \mathcal{R}(\beta)$ or $\frac{c}{b} \mathcal{R}(\alpha) \leq \frac{c}{b} \mathcal{R}(\gamma)$ and by Lemma 3.5

$$
(1+\mathcal{R}(\alpha))(1+\mathcal{R}(\beta))=1+\mathcal{R}(\beta)
$$

or

$$
(1+\mathcal{R}(\alpha))\left(\frac{c}{b}+\frac{c}{b} \mathcal{R}(\gamma)\right)=\frac{c}{b}+\frac{c}{b} \mathcal{R}(\gamma)
$$

Then by (18) we conclude that

$$
\mathcal{R}(\alpha) \leq \max (\mathcal{R}(\beta), \mathcal{R}(\gamma)) \Rightarrow \alpha(\beta+\gamma)=\alpha \beta+\alpha \gamma
$$

From (21) and (22) we obtain

$$
\begin{aligned}
\mathcal{R}(\alpha) & \leq \max (\mathcal{R}(\beta), \mathcal{R}(\gamma)) \vee(\beta+\gamma) A=A \max (|\beta|,|\gamma|) \\
& \Rightarrow \alpha(\beta+\gamma)=\alpha \beta+\alpha \gamma .
\end{aligned}
$$

We conclude by combining (20) and (23).

To complete the proof of Theorem 5.6 for the case of zeroless numbers we need one more lemma. 
Lemma 5.16 If $\beta+\gamma \in \mathcal{N}$ and $\alpha(\beta+\gamma) \neq \alpha \beta+\alpha \gamma$, then $(\beta+\gamma) A \subset A \max$ $(|\beta|,|\gamma|)$.

Proof Suppose that $\alpha(\beta+\gamma) \neq \alpha \beta+\alpha \gamma$. Then, since $\beta+\gamma=B+C$, by Theorem 5.15 we obtain $\mathcal{R}(\alpha)>\max (\mathcal{R}(\beta), \mathcal{R}(\gamma))$. We prove that

$$
\max (\mathcal{R}(\beta), \mathcal{R}(\gamma)) \mathcal{R}(\alpha) \subset \mathcal{R}(\alpha) .
$$

Let $t \in \mathcal{R}(\alpha)$. Because $\mathcal{R}(\alpha) \subseteq \oslash$, one has $t \mathcal{R}(\alpha) \subseteq \mathcal{R}(\alpha)$. Suppose by contradiction that $t \mathcal{R}(\alpha)=\mathcal{R}(\alpha)$. Then $t \in t \mathcal{R}(\alpha)$. Then there exists $x \in \mathcal{R}(\alpha)$ such that $t x=t$. Hence $1 \in \mathcal{R}(\alpha)$. This is in contradiction with the fact that $\mathcal{R}(\alpha) \subseteq \oslash$. Hence $t \mathcal{R}(\alpha) \subset \mathcal{R}(\alpha)$. Applying this inclusion to $t>\max (\mathcal{R}(\beta), \mathcal{R}(\gamma))$ we obtain

$$
\max (\mathcal{R}(\beta), \mathcal{R}(\gamma)) \mathcal{R}(\alpha) \subseteq t \mathcal{R}(\alpha) \subset \mathcal{R}(\alpha) .
$$

This implies that

$$
\left(\frac{B}{b}+\frac{C}{c}\right) \frac{A}{a} \subset \frac{A}{a}
$$

Because $c \leq b$,

$$
\left(\frac{B}{b}+\frac{C}{b}\right) A \subset A
$$

Hence, because $\beta+\gamma=B+C$,

$$
(\beta+\gamma) A \subset A \beta
$$

We conclude that

$$
(\beta+\gamma) A \subset A \max (|\beta|,|\gamma|)
$$

Proof of Theorem 5.6 for zeroless external numbers: If follows immediately from Theorem 5.15 that

$$
\begin{aligned}
\alpha(\beta+\gamma) & =\alpha \beta+\alpha \gamma \\
& \Rightarrow \mathcal{R}(\alpha) \leq \max (\mathcal{R}(\beta), \mathcal{R}(\gamma)) \vee(\beta+\gamma) A=A \max (|\beta|,|\gamma|)
\end{aligned}
$$

If $\beta+\gamma \notin \mathcal{N}$, the reverse implication follows from Theorem 5.15.2. If $\beta+\gamma \in \mathcal{N}$, the reverse implication follows from Theorem 5.15.1 and Lemma 5.16. 


\subsection{Binomial formulae.}

As an application we study the effect of the distributive law on some binomial forms. Let $\alpha=a+A, \beta=b+B$ and $\gamma=c+C$ with $a, b$ and $c$ real numbers and $A, B$ and $C$ neutrices.

Firstly, because $\alpha$ is more precise than $\alpha$, we always have

$$
\alpha(\alpha+\beta)=\alpha^{2}+\alpha \beta \text {. }
$$

Secondly, we investigate the validity of the equality

$$
(\alpha-\beta)(\alpha+\beta)=\alpha^{2}-\beta^{2} .
$$

If $\alpha$ and $\beta$ are neutrices it is easy to verify the equality (25) directly. In the remaining case, we suppose without loss of generality that both $\alpha$ and $\beta$ are non-negative. Then by Theorem 5.8

$$
(\alpha-\beta)(\alpha+\beta)=(\alpha-\beta) \alpha+(\alpha-\beta) \beta
$$

Hence by (24)

$$
(\alpha-\beta) \alpha+(\alpha-\beta) \beta=\alpha^{2}-\alpha \beta+\alpha \beta-\beta^{2}=\alpha^{2}-\beta^{2}+N(\alpha \beta) .
$$

Hence always $(\alpha-\beta)(\alpha+\beta) \supseteq \alpha^{2}-\beta^{2}$. Observe that $N\left(\alpha^{2}-\beta^{2}\right)=\alpha A+\beta B$ and that $N(\alpha \beta)=\alpha B+\beta A$. Hence (25) holds if $\alpha B+\beta A \subseteq \alpha A+\beta B$, say, if $B \leq A$ and $\beta \leq \alpha$.

Thirdly, we show that if $\alpha$ and $\beta$ are neither opposite with respect to $A$ nor to $B$,

$$
(\alpha+\beta)^{2}=\alpha^{2}+2 \alpha \beta+\beta^{2} .
$$

Indeed, by Lemma 5.5.1 the numbers $\alpha$ and $\beta$ are not opposite with respect to $A+B=N(\alpha+\beta)$. Then by Theorem 5.6 and (24)

$$
\begin{aligned}
(\alpha+\beta)^{2} & =(\alpha+\beta)(\alpha+\beta)=\alpha(\alpha+\beta)+\beta(\alpha+\beta) \\
& =\alpha^{2}+\alpha \beta+\beta \alpha+\beta^{2}=\alpha^{2}+2 \alpha \beta+\beta^{2} .
\end{aligned}
$$

Finally we extend the equality (26) to a Binomial Theorem for external numbers. We need some properties of the relative uncertainty.

Lemma 5.17 Let $\alpha=a+A$ and $\beta=b+B$ be external numbers. Then

(1) $\mathcal{R}(\alpha \beta)=\mathcal{R}(\alpha)+\mathcal{R}(\beta)$.

(2) If $k \in \mathbb{N}$ is standard, $\mathcal{R}\left(\alpha^{k}\right)=\mathcal{R}(\alpha)$. 
If $\alpha$ and $\beta$ are zeroless the lemma is an easy consequence of Proposition 4.15.1. Else the equalities are trivially satisfied.

Theorem 5.18 Let $\alpha=a+A$ and $\beta=b+B$ be external numbers. If $\alpha$ and $\beta$ are neither opposite with respect to $A$ nor to $B$, for standard $n \in \mathbb{N}, n \geq 1$

$$
(\alpha+\beta)^{n}=\sum_{k=0}^{n}\left(\begin{array}{l}
n \\
k
\end{array}\right) \alpha^{n-k} \beta^{k} .
$$

The proof is by external induction. If $n=1$, then (27) is clearly true. Suppose that (27) is true for standard $n$. Then

$$
(\alpha+\beta)^{n+1}=(\alpha+\beta)(\alpha+\beta)^{n}=(\alpha+\beta) \sum_{k=0}^{n}\left(\begin{array}{l}
n \\
k
\end{array}\right) \alpha^{n-k} \beta^{k} .
$$

The neutrix $C \equiv N\left(\sum_{k=0}^{n}\left(\begin{array}{l}n \\ k\end{array}\right) \alpha^{n-k} \beta^{k}\right)$ is a sum with a standard finite number of multiples of $A$ and $B$. Hence Lemma 5.5.1 and Lemma 5.5.2 imply that $\alpha$ and $\beta$ are not opposite with respect to $C$. By Theorem 5.6

$$
(\alpha+\beta) \sum_{k=0}^{n}\left(\begin{array}{l}
n \\
k
\end{array}\right) \alpha^{n-k} \beta^{k}=\alpha \sum_{k=0}^{n}\left(\begin{array}{l}
n \\
k
\end{array}\right) \alpha^{n-k} \beta^{k}+\beta \sum_{k=0}^{n}\left(\begin{array}{l}
n \\
k
\end{array}\right) \alpha^{n-k} \beta^{k} .
$$

It follows from Lemma 5.17 that $\mathcal{R}(\alpha) \leq \mathcal{R}\left(\left(\begin{array}{l}n \\ k\end{array}\right) \alpha^{n-k} \beta^{k}\right)$ for all $k$ such that $0 \leq$ $k \leq n-1$, and $\mathcal{R}(\beta) \leq \mathcal{R}\left(\left(\begin{array}{l}n \\ k\end{array}\right) \alpha^{n-k} \beta^{k}\right)$ for all $k$ such that $1 \leq k \leq n$. Repeated application of Theorem 5.6 yields

$$
\begin{aligned}
& \alpha \sum_{k=0}^{n}\left(\begin{array}{l}
n \\
k
\end{array}\right) \alpha^{n-k} \beta^{k}+\beta \sum_{k=0}^{n}\left(\begin{array}{l}
n \\
k
\end{array}\right) \alpha^{n-k} \beta^{k} \\
= & \sum_{k=0}^{n}\left(\begin{array}{l}
n \\
k
\end{array}\right) \alpha^{n+1-k} \beta^{k}+\sum_{k=0}^{n}\left(\begin{array}{l}
n \\
k
\end{array}\right) \alpha^{n-k} \beta^{k+1} .
\end{aligned}
$$

Because the relative uncertainty of natural numbers is zero, again by Theorem 5.6

$$
\sum_{k=0}^{n}\left(\begin{array}{l}
n \\
k
\end{array}\right) \alpha^{n+1-k} \beta^{k}+\sum_{k=0}^{n}\left(\begin{array}{l}
n \\
k
\end{array}\right) \alpha^{n-k} \beta^{k+1}=\sum_{k=0}^{n+1}\left(\begin{array}{c}
n+1 \\
k
\end{array}\right) \alpha^{n+1-k} \beta^{k} .
$$




\section{References}

[1] I.P. van den Berg, M. Diener, Diverses applications du lemme de Robinson en analyse nonstandard, C. R. Ac. Sci. Paris, série I, 293(1981), 501-504.

[2] I.P. van den Berg, Nonstandard Asymptotic Analysis, Springer Lecture Notes in Mathematics 1249 (1987).

[3] I.P. van den Berg, A decomposition theorem for neutrices, Annals of Pure and Applied Logic, 161:7(2010), 851-865; doi:10.1016/j.apal.2009.10.003.

[4] N.G. de Bruijn, Asymptotic Analysis, North Holland, 1961.

[5] U.L. Clotz, Untersuchungen einiger topologischer Sachverhalte und Konstruktionen in HST, PhD thesis, Bergische Universität Wuppertal, 2009; available at http://elpub.bib.uniwuppertal.de/servlets/DerivateServlet/Derivate-968/dc0901.pdf.

[6] J.G. van der Corput, Introduction to the neutrix calculus, J. Analyse Math. 7(1959/1960), 281-399.

[7] F. and M. Diener (eds.), Nonstandard Analysis in Practice, Springer Universitext, 1995.

[8] F. Diener, G. Reeb, Analyse Non Standard, Hermann, Paris, 1989.

[9] H. Gonshor, Remarks on the Dedekind completion of a nonstandard model of the reals, Pacific J. Math. 118(1)(1985), 117-132.

[10] E. R. Hansen, A Generalized Interval Arithmetic, Springer Lecture Notes in Computer Science 29 (1975).

[11] K. Hrbáček, Axiomatic foundations for nonstandard analysis, Fund. Math. 98(1)(1978), $1-19$.

[12] K. Hrbáček, Realism, nonstandard set theory, and large cardinals, Ann. Pure Appl. Logic 109 (1-2)(2001), 15-48; doi:10.1016/S0168-0072(01)00039-2.

[13] V. Kanovei, M. Reeken, Mathematics in a nonstandard world I, Mathematica Japonica 45, no. 2(1997), 369-408.

[14] V. Kanovei, M. Reeken, Nonstandard Analysis, Axiomatically, Springer Monographs in Mathematics, 2004.

[15] H.J. Keisler, J.H. Schmerl, Making the hyperreal line both saturated and complete, J. Symbolic Logic 56(1991), 1016-1025; doi:10.2178/js1/1183743748.

[16] F. Koudjeti, Elements of External Calculus with an aplication to Mathematical Finance, $\mathrm{PhD}$ thesis, Labyrinth publications, Capelle a/d IJssel, The Netherlands, 1995.

[17] F. Koudjeti, I.P. van den Berg, Neutrices, external numbers and external calculus, in: Nonstandard Analysis in Practice (eds. F. and M. Diener), Springer Universitext 1995, 145-170.

[18] R. E. Moore, R. B. Kearfott and M. J. Cloud, Introduction to Interval Analysis, SIAM, 2009. 
[19] E. Nelson, Internal Set Theory, an axiomatic approach to nonstandard analysis, Bull. Am. Math. Soc., 83(6)(1977), 1165-1198; doi:10.1090/S0002-9904-1977-14398-X.

[20] A. Robinson, E. Zakon, A set-theoretical characterization of enlargements, in Applications of Model Theory to Algebra, Analysis, and Probability (ed. W. A. J. Luxemburg), Holt, Rinehart and Winston, 1969, 109 - 122.

[21] F. Wattenberg, [0, $\infty$-valued, translation invariant measures on $\mathbb{N}$ and the Dedekind completion of $* \mathbb{R}$, Pacific J. Math., 90(1980), 223-247.

[22] L. A. Zadeh, Fuzzy sets, Information and Control 8 (1965), 338-353.

[23] E. Zakon, A new variant of non-standard analysis, in Victoria Symposium on Nonstandard Analysis(1972)(ed. A. Hurd), Lecture Notes in Mathematics 369, Springer-Verlag, 1974, 313-339.

Department of Mathematics, University of Évora, Portugal

bruno.salsa@gmail.com, ivdb@uevora.pt

Received: 13 May $2010 \quad$ Revised: 26 March 2011 